\title{
Efeito de duas estratégias educativas em saúde no autocuidado de usuários de um centro de atenção psicossocial
}

\author{
Effect of two educational health strategies on the self-care of users of a psychosocial care \\ center
}
Efecto de dos estrategias educativas de salud en el autocuidado de los usuarios de un centro de atención psicosocial.

Cláudia Cristina Rolim da Silva ${ }^{1 *}$, Célio Fernando de Sousa Rodrigues².

\section{RESUMO}

Objetivo: Comparar os efeitos de duas estratégias educativas em saúde e suas relações nas ações de autocuidado de pessoas acompanhadas por uso de álcool e outras drogas. Métodos: Trata-se de um estudo quantitativo, analítico, descritivo, experimental, com grupo controle não-equivalente desenvolvido através da avaliação da capacidade de autocuidado e comportamento de uso de droga após a aplicação de intervenções educativas seguindo o modelo tradicional ou dialógico de pessoas acompanhadas em Centro de Atenção Psicossocial Álcool e outras Drogas. Para análise dos dados foram utilizados o teste de Friedman e o coeficiente de correlação de Pearson. Resultados: Foi demonstrado que os efeitos no comportamento do uso de etanol e o autocuidado do grupo com estratégia dialógica apresentaram melhores resultados e quanto melhor o autocuidado, maior é a possibilidade de redução da ingestão de álcool. Conclusão: As estratégias educativas utilizadas influenciaram as ações de autocuidado e a redução do consumo de etanol e maconha.

Palavras-chave: Educação em saúde, Autocuidado, Transtornos relacionados ao uso de substâncias, Saúde mental.

\begin{abstract}
Objective: Compare the effects of two educational strategies in health care and the relation between actions of self-care of individuals who consumed alcohol and other drugs. Methods: This is a study quantitative, analytic, descriptive, experimental com a group control non-equivalent, developed through an evaluation of the capacity of self-care and behavior of drug consumption after the application of educational interventions and following the traditional or dialogical models applied to people who were being analyzed in Center for Psychosocial attention, alcohol and other drugs. Pearson Correlation Coefficient and the Friedman test were used to analyze and measure data. Results: It has been shown that the behavior effect under the influence of Ethanol and self-care management in the group where the dialogical strategy was applied presented better results and the greater the self-care, greater is the possibility of reduction of alcohol ingestion. Conclusion: The applied educational strategies influenced the performance of self-care and the reduction of Ethanol and Marijuana consumption.
\end{abstract}

Keywords: Health education, Self-care, Substance related disorders, Mental health.

1 Universidade Estadual de Ciências da Saúde de Alagoas (UNCISAL), Maceió - AL.

*E-mail: clarolimsilva@gmail.com

2 Universidade Federal de Alagoas (UFAL), Maceió - AL. 


\section{RESUMEN}

Objetivo: Compare los efectos de dos estrategias educativas de salud y sus relaciones en las acciones de autocuidado para personas acompañadas del uso de alcohol y otras drogas. Métodos: Este es un estudio cuantitativo, analítico, descriptivo, experimental, con un grupo de control no equivalente desarrollado a través de la evaluación de la capacidad de autocuidado y el comportamiento de consumo de drogas después de la aplicación de intervenciones educativas siguiendo el modelo tradicional o dialógico de personas monitoreadas en el Centro de Atención. Alcohol psicosocial y otras drogas. Para el análisis de datos, se utilizaron la prueba de Friedman y el coeficiente de correlación de Pearson. Resultados: Se ha demostrado que los efectos sobre el comportamiento del uso de etanol y el autocuidado del grupo con una estrategia dialógica mostraron mejores resultados y cuanto mejor es el autocuidado, mayor es la posibilidad de reducir el consumo de alcohol. Conclusión: Las estrategias educativas utilizadas influyeron en las acciones de autocuidado y en la reducción del consumo de etanol y marihuana.

Palabras clave: Educación en salud, Autocuidado, Trastornos relacionados con sustancias, Salud mental.

\section{INTRODUÇÃO}

É de se considerar os fatores existentes e a tamanha complexidade nos diversos âmbitos psicossociais quando se remete ao contexto de uso de substâncias psicoativas, a maior parte dos pesquisadores discorrem que a dependência química é uma doença crônica e recorrente que acomete o sistema nervoso central (SNC) ocasionada pelo desejo excessivo e de modo não controlado pela droga, estes prejuízos são decorrentes pelo consumo e recorrentes, de modo que os próprios indivíduos já os identificam em determinado momento, apesar dos prejuízos decorrentes e já instalados e observados até pelo próprio indivíduo (MALBERGIER A e OLIVEIRA JR H, 2011).

Isto é preocupante, uma vez que em muitas situações os indivíduos priorizam o consumo, seja de álcool ou outras drogas ilícitas em detrimento a obtenção de alimentos, do convívio familiar e autocuidado, acarretando em conflitos, em discussões, no surgimento de doenças, deficit de memória, tomada de decisões, capacidade de absorver informações, e na violência conjugal (MONTEIRO SFS, et al., 2011; RIGONI MS, et al., 2008).

Para o atendimento dessas situações, instituiu-se uma rede de atenção psicossocial para pessoas com sofrimento mental (RAPS) e ou por uso de álcool e outras drogas. Atualmente a RAPS é composta pelas unidades básicas, hospitais gerais, unidades de acolhimento, serviços de urgência, serviços de residência terapêutica, hospital psiquiátrico e Centros de Atenção Psicossocial (CAPS) (BRASIL, 2019).

Todos os profissionais que atuam nos CAPS Ads trabalham de modo interdisciplinar seguindo os preceitos da humanização da assistência e a valorização da singularidade (ROCHA THR, et al., 2019). Entretanto, para Oliveira EN, et al. (2019) é essencial que nesses serviços ocorra um aumento da frequência da realização de grupos educativos em saúde que busquem o desenvolvimento de habilidades em prol do autocuidado, já que pode ocasionar em resultados positivos para os sujeitos.

Para Santos HA, et al. (2018) se as práticas educativas em saúde, forem bem aplicadas, o profissional tem a oportunidade de transmitir ou discutir conhecimentos para o desenvolvimento da autonomia, autocuidado, resgatar a qualidade de vida, prevenir doenças e agravos dos participantes. As quais favorecem o despertar, a obtenção do conhecimento para uma compreensão das situações vivenciadas e a adoção de novos hábitos de vida. Porém, observa-se que essas ações realizadas em geral, estão centradas na doença com a utilização de metodologias tradicionais, de modo que a cultura das pessoas não é considerada como ponto de partida para o planejamento dessas ações (SALCI MA, et al., 2013).

Nesta perspectiva educativa, Dorothea Oren, defende em sua teoria do déficit de autocuidado que o autocuidado é uma ação ensinada e aprendida quando o mesmo tem interesse de resgatar sua saúde e bemestar (QUEIROS PJP, et al., 2014), 
Apesar de reconhecer que as ações individuais possam ser influenciadas por outros fatores como idade, sexo, padrões de vida, sociais, culturais e financeiros (OREM DE e TAYLOR SG, 2011). Como também pelos hábitos relacionados ao padrão de uso de drogas, uma vez que geralmente as relações sociais e culturais são construídas e prejudicadas devido ao uso descontrolado e abusivo.

Diante destes pressupostos, o conhecimento desses resultados poderá inicialmente trazer subsídios para uma restruturação de posturas e de modelos educacionais ainda prevalentes, como também promover reflexões de ações, no sentido de uma prática educativa que promova saúde de pessoas e da população (FIGUEIREDO MFS, et al., 2010).

Dessa forma, o objetivo deste trabalho é comparar os efeitos de duas estratégias educativas em saúde e suas relações nas ações de autocuidado de pessoas acompanhadas por uso de álcool e outras drogas, identificando o impacto dessas estratégias utilizadas na redução do consumo de etanol e maconha.

\section{MÉTODOS}

Trata-se de um estudo analítico, descritivo, experimental com grupo controle não-equivalente. A população deste estudo é composta por usuários acompanhados em um CAPS AD. Foram recrutados por conveniência, usuários que estavam em tratamento diário, três dias, duas vezes por semana ou com indicação para acompanhamento pontual no grupo de autocuidado (1x por semana), na etapa da aplicação das intervenções.

A alocação dos participantes em cada grupo ocorreu a partir da pontuação gerada na escala de autocuidado - ASA-A, a partir da categorização dos dados proposta por Silva JVS, et al (2012), que classificaram o autocuidado dos participantes de seu estudo como péssimo, (24 a 40 pontos); ruim (40 a 56 pontos); regular (56 a 72 pontos); boa (72 a 88 pontos); muito boa (88 a 104 pontos) e ótima (104 a 120 pontos).

A partir dessa classificação, os mesmos foram alocados em cada grupo (GT ou GD). Buscou-se a divisão por grupo de tratamento de 1:1 conforme os participantes iriam sendo admitidos durante a pesquisa. Este estudo, seguiu-se a composição total de participantes ( $n-40)$, divididos em dois

Grupos, experimental e controle, utilizada por Zarshenas L, et al. (2017), com homens internados em um hospital psiquiátrico devido ao uso de entorpecentes. Os tipos de drogas não foram considerados como método de agrupamento, por que a estratégia de divisão por tipo de droga não é utilizada pelos profissionais que trabalham com essa população. $E$ dar ênfase ao objeto droga não permite a visualização e consequentemente intervenção profissional diante das situações e conflitos nos aspectos sociais, culturais, cognitivos e afetivos, que são apontadas como causas e ou consequências do consumo e que provocam o relacionamento dessas pessoas com as substâncias (MARTINS JS e MACRAE E, 2010; SOUZA SEF, et al., 2017).

Os critérios de inclusão foram pessoas dependentes de substâncias psicoativas, cadastradas no CAPS $A D$, que estivessem lúcidas e orientadas no tempo e espaço com idade igual ou superior a 18 anos, com disponibilidade para participação de grupos educativos, entrevistas de acompanhamento e intervenções individuais no período de 3 meses. Os critérios de exclusão foram pessoas que não participaram de nenhum grupo educativo, que apresentassem cognição prejudicada observada pelo pesquisador diante da entrevista e que desejassem sua exclusão durante a execução pesquisa.

Foram aplicados durante o pré-teste e os pós testes a Escala de autocuidado, Appraisal of Self-care Agency-ASA-ESCALE (ASA-A) validada por Silva JV e Kimura, M (2012) e Silva JV e Domingues EAR (2017). Outro instrumento que também possibilitou a obtenção de informações em relação ao consumo de álcool e outras drogas, sobre os aspectos sociais, familiares e financeiros foi aplicado pela pesquisadora, utilizando como referencial teórico para sua elaboração instrumentos de triagem de uso de álcool e ou outras drogas: O ASSIT, CAGE e o AUDIT (BRASÍLIA, 2014).

As intervenções educativas foram conduzidas pela pesquisadora principal, no formato individual e grupos. O seu planejamento e execução foram elaboradas seguindo as definições da pedagogia Freiriana que com 
suas contribuições, aponta as diferenças existentes entre os modelos tradicional, conceituado como bancário e o modelo dialógico que considera a realidade dos sujeitos, proporcionando uma construção coletiva do conhecimento.

A quantidade de intervenções/estratégias educativas aplicadas no formato de grupos foram vinte e oito ( $\mathrm{n}$ 28), subdivididos com cada proposta educativa, e ocorreram de outubro de 2018 a março de 2019. As variáveis analisadas foram a capacidade de autocuidado dos participantes (antes e depois) e a frequência e quantidade do consumo de drogas (transformado em unidades/doses/mês).

Os dados foram tabelados e armazenados no programa Excel. Os dados quantitativos foram apresentados na forma de média aritmética e frequência. Os dados qualitativos foram apresentados na forma de quadro e frequência. $\mathrm{Na}$ análise estatística inferencial, para comparar os grupos de acordo com os escores foi utilizado estatística comparativa utilizando as medidas de tendência central.

O consumo de etanol foi calculado considerando o tipo de bebida, a quantidade (medida e volume) e o consumo por mês, referido pelos participantes, utilizando a calculadora online disponível na BVS, (S/ANO) resultando-se em unidades/dose por mês.

A partir dos dados obtidos, foi utilizado o teste de Friedman para avaliar o consumo de etanol de ambos os grupos e para os efeitos do autocuidado no consumo de etanol foi utilizado o coeficiente de correlação de Pearson. O programa Bioestat - 5.0 foi utilizado para obtenção dos indicadores, considerando o valor significante quando $p<0,05$.

O presente artigo se compõe de uma parte de uma tese de mestrado intitulada "Comparativo de duas estratégias educativas em saúde e suas relações nas ações do autocuidado de usuários de álcool e outras drogas".

O estudo foi desenvolvido conforme o que preconiza a resolução $n^{\circ} 466$, de 12 de dezembro de 2012, do Conselho Nacional de saúde, que discorre sobre as diretrizes e normas envolvendo seres humanos. E foi realizado, após a aprovação no comitê de ética, número do parecer: 2.768 .354 e CAAE: 89195918.6.0000.5011.

\section{RESULTADOS E DISCUSSÃO}

\section{Perfil dos participantes}

Do total dos participantes deste estudo ( $n-40)$ todos são do sexo masculino, isto reflete a realidade dos locais de tratamento para indivíduos com transtornos decorrentes por uso de drogas, onde há uma prevalência de homens (ZARSHENAS L, et al., 2017; BOGENSCHUTZ MP, et al., 2015; DIEPERINK E, et al., 2014). Uma das causas para este comportamento é que eles são mais acometidos pela doença, os hábitos sociais do consumo são mais elevados, e por isto buscam mais a assistência (FARIA JG e SCHNEIDER DR 2009).

Em relação a idade, no grupo tradicional a idade média dos participantes foi de 46,7 anos (mínimo de 20 anos e máximo de 72 anos), no grupo de intervenção dialógica a idade média dos participantes foi de 42,4 anos (mínimo de 19 anos e máximo de 59 anos). Almeida RA, et al. (2014) verificaram diferenças em relação a idade média de seus participantes, de 36,84 anos. Embora tenha ocorrido semelhanças deste com outros estudos, de 45, 4 anos (DAWSON-ROSE C, et al., 2017). Observa-se então, que a maioria de usuários estão na faixa de idade superior a 36 anos.

Quanto ao estado civil, no grupo tradicional, a maioria foi composta por casados (45\%) afirmaram ser católicos (55\%), com escolaridade de ensino fundamental incompleto (65\%). Enquanto que no grupo dialógico, a maior parte estavam separados $(45 \%)$ e referiram ser católicos $(60 \%)$, com escolaridade de ensino fundamental incompleto $(50 \%)$.

Quanto ao tipo de droga, a substância etílica isoladamente, foi referida por $67,5 \%$ das pessoas, mas quando associado com outros tipos de drogas, $17,5 \%$ dos entrevistados, referiram usar também a maconha; 
10\%; o tabaco; $2,5 \%$ inalante (solvente) e 2,5\% crack. Apesar de lícita (permitida por lei), o álcool é a primeira e principal droga consumida no Brasil. Dados da Organização Mundial da Saúde, OMS (2018) apontam que $40 \%$ dos brasileiros fizeram o consumo de álcool no último ano, deste percentual, $54 \%$ eram homens. Informações obtidas em uma pesquisa nacional, realizada com adolescentes nos Estados Unidos, foram semelhantes ao encontrado neste estudo, pois os autores Barry AE, et al, (2016) observaram que o etanol é a substância mais prevalente e também é a porta de entrada para o uso de outras drogas (maconha e tabaco).

\section{Consumo de etanol e maconha relatado}

Para a avaliação da quantidade de consumo relatada de etanol ingerida os participantes que afirmaram não ter controle foi considerado unidade/dose máxima de 1710 unidades, doses/ mês.

Considerando tais pressupostos, no período estudado, quanto ao consumo de etanol auto relatado, houve elevação do consumo em ambos os grupos no $2^{\circ}$ mês de tratamento, embora os participantes do grupo $D$ reduziram de modo mais efetivo no $3^{\circ}$ mês de tratamento (Gráfico 1 ).

Gráfico 1 - Comparativo do consumo médio mensal de etanol relatado dos grupos:

Tradicional (GT) e dialógico (GD).

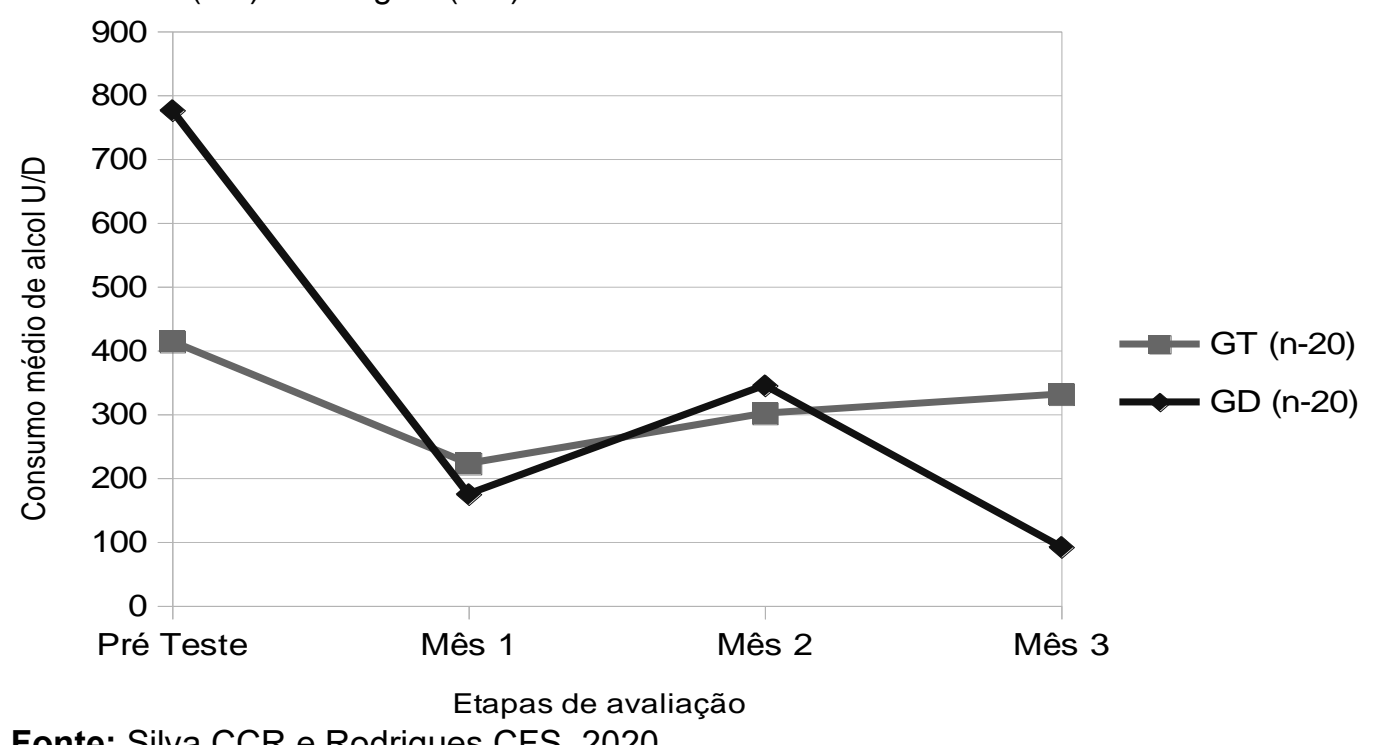

Fonte: Silva CCR e Rodrigues CFS, 2020.

Verifica-se então uma redução no consumo de álcool no grupo $D(p=0,001)$ quando comparado com o grupo $T(p=0,65)$. No geral, o consumo do etanol em relação ao pré teste de ambos os grupos demonstrou um efeito na redução estatisticamente significativo do grupo exposto a estratégia educativa dialógica $(p<$ $0,05)$.

O comportamento distinto dos usuários diante da ingestão de álcool dos participantes em ambos os grupos, apontam que o modelo dialógico tem maior eficácia para uma mudança de atitudes, e por conseguinte para a redução do consumo de substância. Isto se dá por que ao aplicar a estratégia dialógica, na educação em saúde, os profissionais despertam o senso crítico e o desenvolvimento da autonomia, de habilidades sociais e pessoais para a diminuição de hábitos/posturas que prejudicam a saúde das pessoas envolvidas (SOUZA LPS, et al., 2011).

Conforme verificado, os dados apontam que apesar da estratégia educativa tradicional produzir efeitos menores quando comparado ao método dialógico, ela consegue ser melhor que uma intervenção breve. Pesquisadores da área discorrem atualmente que intervenções educativas repetidas e que favoreçam o acompanhamento pelos profissionais de saúde de modo mais frequente trazem mais sucesso para redução de consumo de álcool do que ações pontuais (GLASS JE, et al., 2017).

Segundo os autores supracitados, as novas evidências científicas apontam que o modelo de intervenção breve bastante difundido anteriormente, precisa de modificações, de forma que aconteça repetições ao longo 
do tempo, e que não seja exclusivo para os serviços de atenção primária, mas que se estenda para outros espaços especializados, os quais assistem pessoas com transtornos graves pelo uso de entorpecentes.

Em relação aos prejuízos decorrentes do consumo, dos indivíduos avaliados neste estudo, observou-se que ao longo das intervenções as percepções foram sendo modificadas, para o reconhecimento dos malefícios advindos com o uso. Fato, também identificado por outros pesquisadores (FROTA GAS, et al., 2018; GABATZ RIB, et al., 2013). Porém, durante o presente estudo, foi notado ainda que a maioria dos participantes, que afirmaram o consumo concomitante de duas drogas, não demonstraram inicialmente preocupação em relação ao uso combinado durante a entrevista e grupos realizados.

Pressupõe-se que essa indiferença pode estar associada ao tipo de substância mais referida, em conjunto com o etanol pelos participantes (maconha), presente em $(17,5 \%)$ dessa amostra, esta é a principal droga ilícita mais consumida no mundo, em 2017 aproximadamente 188 milhões de indivíduos referiram ter feito o uso (UNODC, 2019)

Segundo Gentil V (2019) outra causa é que os efeitos deletérios nem sempre são percebidos por essa população, como as questões relacionados a cognição, ao aprendizado, memória e tomada de decisão. Estudos recentes com adolescentes demonstraram que a maconha causa mais danos cognitivos do que o consumo do álcool, e a longo prazo a maconha pode ainda provocar casos de psicoses (MORIN JG, et al., 2019).

Ainda sobre o consumo de entorpecentes, estão dispostos a frequência de cigarros de maconha autorrelatados consumidos no mês pelos participantes avaliados durante o período estudado (Gráfico 2).

Gráfico 2 - Média de cigarros de maconha/mês fumados por grupo GT e GD.

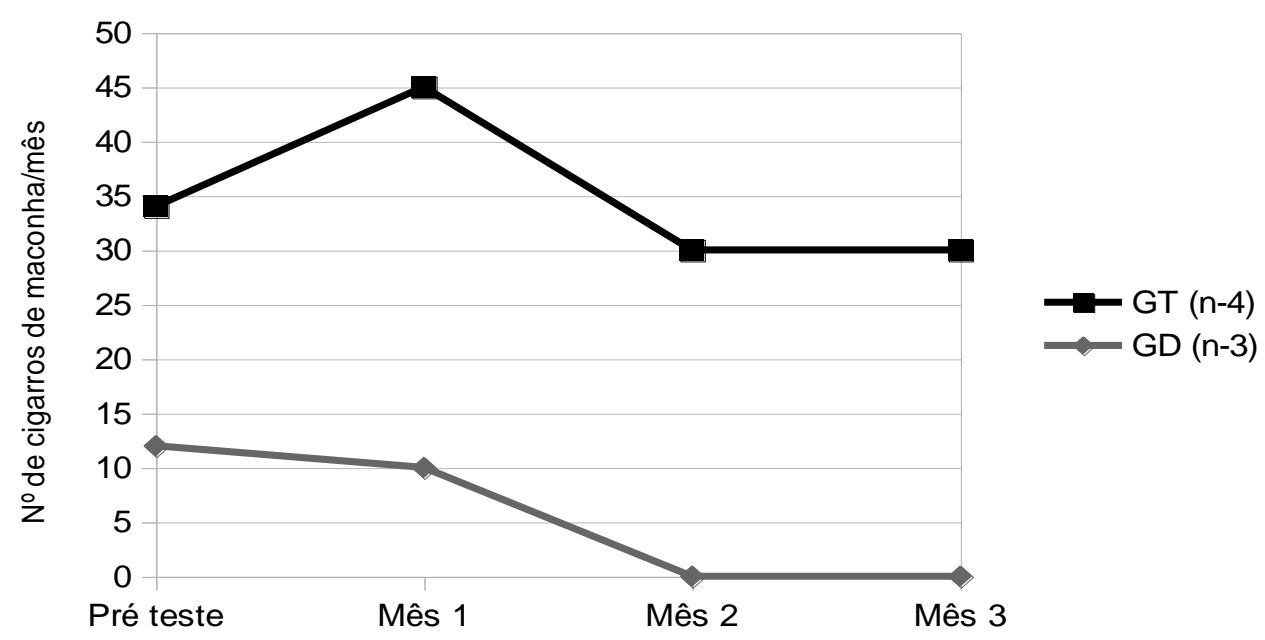

Fonte: Silva CCR e Rodrigues CFS, 2020.

Apesar das diferenças encontradas em relação ao consumo no pré teste, de cigarros de maconha, ao longo das intervenções, o consumo médio de cigarros de maconha por mês, foram reduzidos nas duas intervenções, embora resultados mais expressivos, foram encontrados mais especificamente no grupo $D$, onde ocorreu a interrupção.

É oportuno destacar que o resultado obtido, (grupo D), apesar de vantajosa, não é e nunca será o objetivo final a ser obtido com a proposta dialógica. Mas sim o principal benefício e o mais importante é o processo de transformação das relações desses sujeitos com essas substâncias. Nesse aspecto, Lima EH et al (2018) reforçam que a dialogicidade grupal não está no campo da abstinência, embora não a exclua. Para os autores, é necessário, que o profissional, ao aplicar a educação em saúde amplie seu campo de visão para aumentar a liberdade e a corresponsabilização destes indivíduos frente a seu contexto de vida, nos determinantes e condicionantes dos processos de saúde e doença vivenciados. 
De acordo com esta recomendação, nos indivíduos incluídos no grupo $\mathrm{D}$, esse incentivo para o olhar voltado para realidade, reflexão, transformação, foi executado de modo mais evidente que no grupo T. A interrupção do consumo ocorreu no período estudado, possivelmente em decorrência do desejo de mudança a partir da tomada da consciência e do método educativo aplicado.

\section{Intervenções Educativas e os Reflexos no Autocuidado}

O reconhecimento dos prejuízos relacionados ao consumo dessas substâncias não é algo tão fácil, e requer estratégias para promover o autoconhecimento e o incentivo de mudança de hábitos para um melhor autocuidado e comportamento pela busca de sua saúde.

Não houve diferença estatisticamente significativa no $G T(p=0,50)$ e no $G D(p=0,21)$. No entanto, conforme observa-se no gráfico pelo acompanhamento das médias de autocuidado antes e após da intervenção nos grupos houve um avanço de 5,4 pontos no GD e 0,84 pontos no GT.

Identifica-se ainda um aumento em relação semelhante nos dois grupos, no primeiro $\left(1^{\circ}\right)$ mês das intervenções educativas. No entanto, a partir do $2^{\circ}$ mês de intervenção houve um leve declínio na média de ambos os grupos, e no $3^{\circ}$ mês, o grupo $D$ avançou na média de autocuidado em relação ao grupo $T$, que declinou (Gráfico 3).

Gráfico 3 - Comparação da média de autocuidado dos participantes de ambos os grupos observada após as ações educativas.

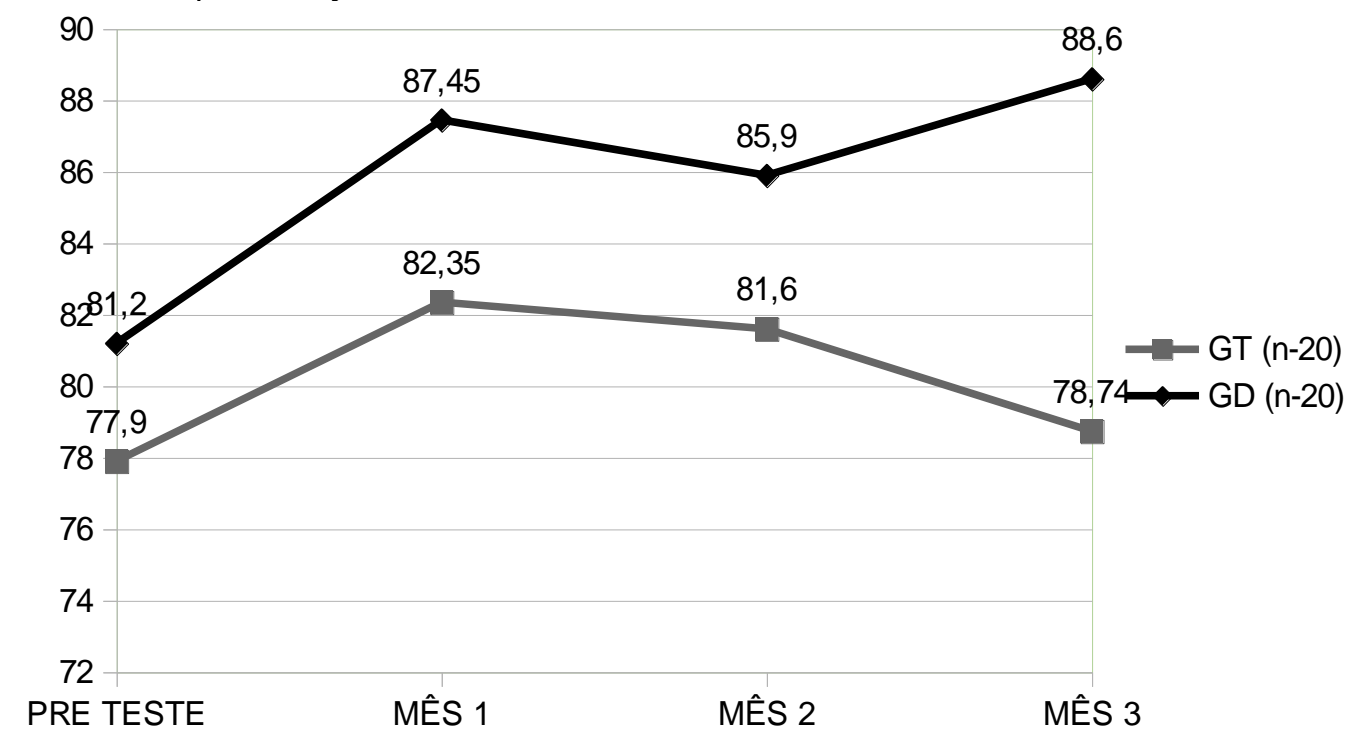

Fonte: Silva CCR e Rodrigues CFS, 2020.

Os dados demonstram que ações de intervenção seguindo o modelo dialógico, durante o período pesquisado, favoreceu de modo mais duradouro e uma maior pontuação, consequentemente na melhoria do autocuidado quando comparado ao grupo tradicional.

Fato também identificado na pesquisa de Vasconcelos SC et al, (2013), que sob a ótica dos usuários de um CAPS AD após a condução de estratégias educativas horizontais, embasadas no respeito, na liberdade e autonomia dos usuários em tratamento. Os mesmos avaliaram positivamente a ação e afirmaram que a participação proporcionou reflexões importantes sobre os hábitos danosos e foram capazes de favorecer a compreensão sobre a importância de reduzir os danos para prevenir doenças.

Nóbrega MPSS (2012) defende que a conduta profissional mais assertiva parte do princípio de motivar pessoas para mudança de comportamentos a partir das necessidades identificadas pelos próprios usuários, com respeito aos seus desejos e singularidades. Ou seja, a decisão de mudar é do indivíduo, o profissional de saúde tem o papel de facilitar esse processo de autopercepção e recuperação de sua saúde. 
Ainda em relação ao gráfico 3 , é observado através das médias, que o comportamento dos participantes do grupo T em relação ao autocuidado avançou após o tratamento ofertado e seguiu com um comportamento semelhante ao grupo $D$ até o segundo mês. No terceiro mês houve um declínio e os grupos seguiram movimentos opostos, isso pode ser explicado, em grande medida, pelo vínculo e pelo despertar de um senso crítico, propostos aos participantes durante a condução dos encontros no grupo D. Uma vez que os grupos eram realizados no mesmo espaço e com os mesmos recursos profissionais, seguindo estratégias educativas diferenciadas.

Este vínculo pode estar associado a participação dos usuários na metodologia, que no grupo $D$, o qual acontece ativamente desde o princípio, já no grupo T o usuário é passivo de uma metodologia já estabelecida pelo profissional responsável.

\section{O Autocuidado e o consumo de álcool}

Os dados obtidos demonstram uma correlação negativa entre as variáveis: consumo $(Y)$ e autocuidado (X), assim, quando uma variável cresce a outra diminui e vice-versa. Desta forma, é possível afirmar que há uma correlação inversa entre a quantidade ingerida de álcool e o autocuidado dos participantes avaliados nesse estudo. Isto é demonstrado através do coeficiente de correlação de Pearson (P de Pearson). (IC- 95\%) (Gráficos 4 e 5).

Gráfico 4 - Correlação de quantidade de álcool ingerida e o autocuidado no grupo GT.

\section{Função consumo/Autocuidado grupo $T$}

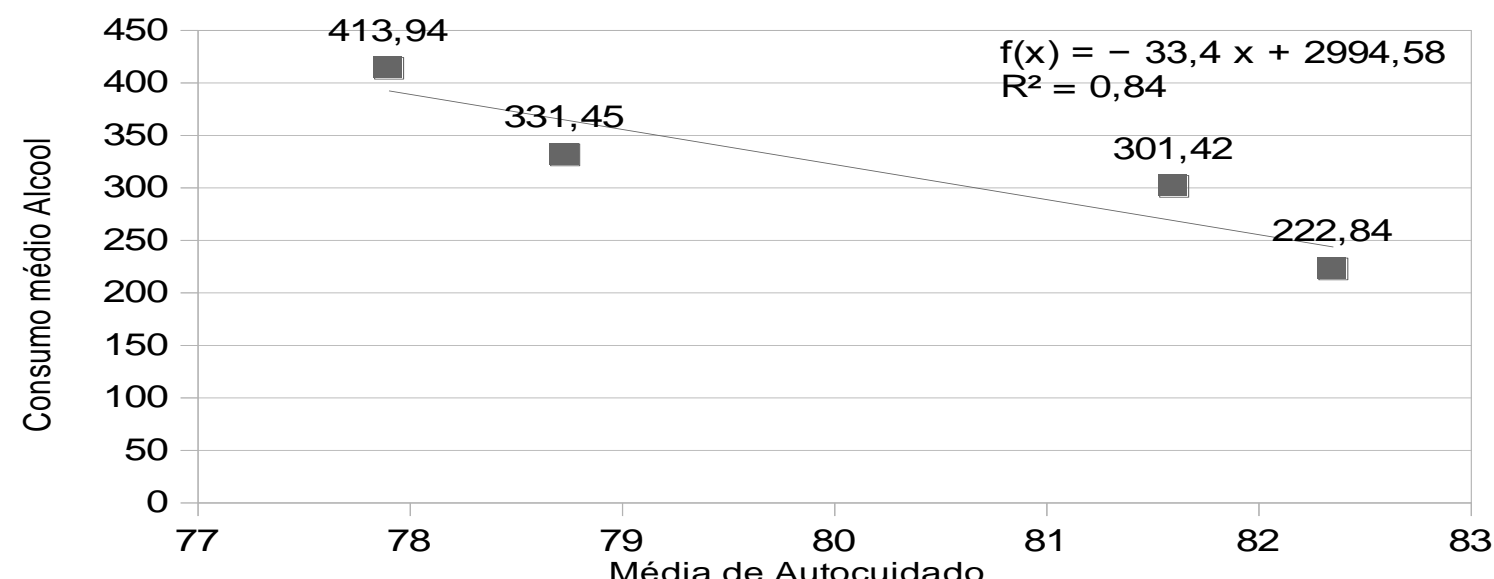

Legenda: Curva de regressão linear Grupo Tradicional (GT). Fonte: Silva CCR e Rodrigues CFS, 2020.

Gráfico 5 - Correlação de quantidade de álcool ingerida e o autocuidado no grupo GD.

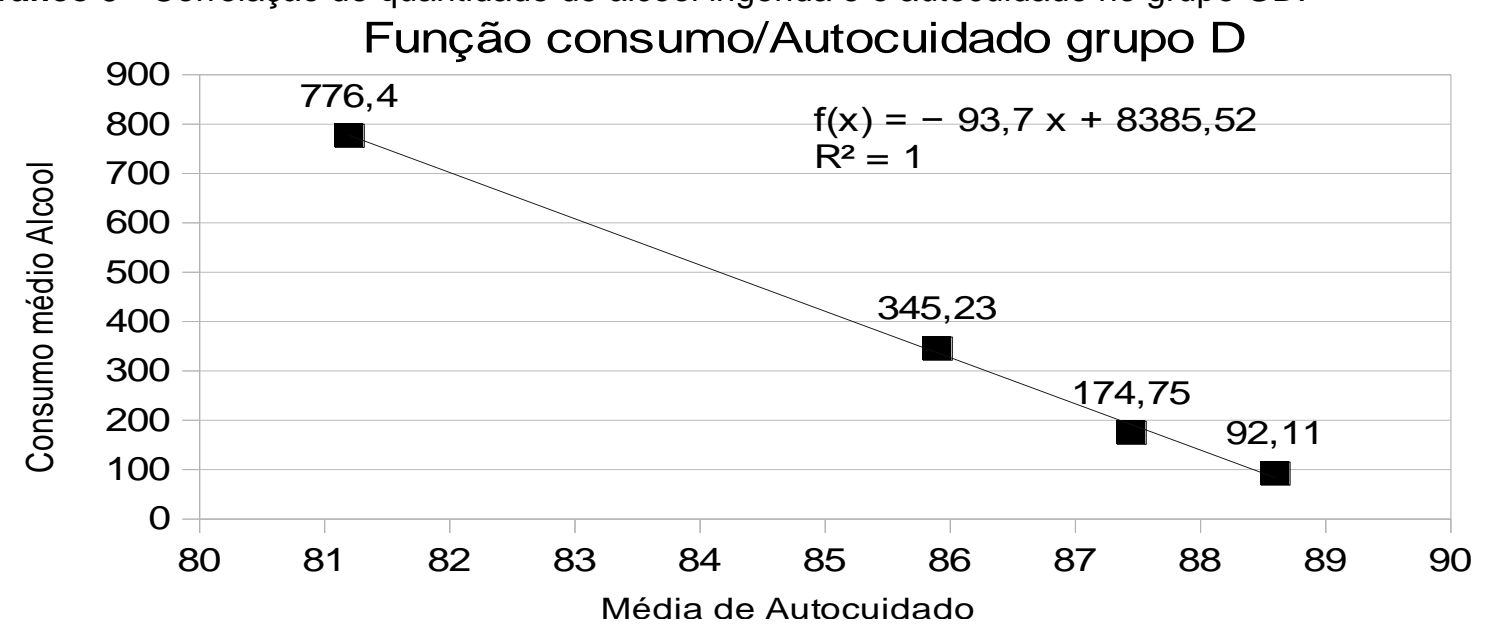

Legenda: Curva de regressão linear Grupo Dialógico (GD). Fonte: Silva CCR e Rodrigues CFS, 2020. 
No grupo tradicional a correlação autocuidado/consumo de álcool obteve um $\mathrm{R}$ de Pearson de $-0,926$ já no grupo dialógico um $R$ de Pearson de -0.998 , quando $R=1$ ou -1 Significa uma correlação perfeita positiva ou negativa entre as duas variáveis. $O$ valor do $\mathrm{R}$ de Pearson acima de 0,9 indica uma correlação muito forte, quase perfeita, demonstrando que o tratamento que obtiver efeito na evolução do autocuidado, por consequência garantirá uma redução do consumo.

Na curva de regressão linear $f(x)=-93,7 x+8385,52=0$, temos um valor ajustado de $x=89,49$, ou seja, para que a metodologia dialógica consiga o sucesso de eliminar o consumo da droga, deve-se buscar uma evolução do autocuidado a pelo menos 89,49 que é o ponto zero da função, onde a linha de regressão toca o eixo $(X)$, e $(Y)$ é igual a zero. Supõe-se que, respeitando a margem de erro, o usuário que alcançar um $X \geq$ 89,49 interromperá o consumo de álcool quando submetido a metodologia dialógica.

Em relação ao grupo tradicional, a curva de regressão linear $F(x)=-33,4 x+2994,58=0$, temos um valor ajustado de $x=89,65$ do mesmo modo, respeitando a margem de erro, o usuário que alcançar um $X \geq 89,65$ interromperá o consumo de álcool quando submetido a metodologia tradicional. Nota-se que, comparando as duas metodologias, a pontuação necessária de autocuidado para a interrupção do consumo foi um pouco menor na metodologia dialógica. O que demonstra uma pequena vantagem em termos de eficiência desta metodologia no tratamento do alcoolismo.

Tal eficiência é salientada quando se compara as inclinações das curvas das duas equações. Percebe-se que a inclinação da curva do grupo dialógico é bem mais acentuada que a do grupo tradicional. A declividade da curva é interpretada como a taxa de variação da variável $(Y)$ em relação à variável $(X)$. Logo, se a taxa de declividade for alta, demonstra que uma variação na pontuação do autocuidado refletirá em uma maior variação na redução da pontuação do consumo.

\section{CONCLUSÃO}

A estratégia dialógica ocasiona em melhor redução no consumo de etanol e maconha. Não houve melhora estatisticamente significativa no autocuidado dos participantes de ambos os grupos. Apesar disto, esteve intimamente relacionado ao consumo do etanol, ou seja, quanto melhor o autocuidado, maior é a possibilidade de redução da ingestão de álcool. Portanto, torna-se importante a aplicabilidade de estratégias educativas dialógicas nos diversos setores de saúde, e principalmente com dependentes químicos com enfoque para 0 incentivo ao autocuidado.

\section{REFERÊNCIAS}

1. ALMEIDA RA, et al. Perfil dos usuários de substâncias psicoativas de João Pessoa. Saúde Debate, 2014. 38(102): 526-538.

2. BARRY AE, et al. Alcohol Marketing on Twitter and Instagram: Evidence of Directly Advertising toYouth/Adolescents. Alcohol and Alcoholism, 2016. 51 (4): 487-492.

3. BRASIL, Ministério da Saúde. Nota Técnica no 11/2019 GGMAD/DAPES/SAS/MS. Esclarecimentos sobre as mudanças na Política Nacional de Saúde Mental e nas Diretrizes da Política Nacional sobre Drogas. Brasília, fev.2019.

4. BRASÍLIA, Secretaria Nacional de Políticas sobre Drogas - SENAD. SUPERA (Sistema para Detecção do Uso Abusivo e Dependência de Substâncias Psicoativas; Encaminhamento, Intervenção Breve, Reinserção Social e Acompanhamento. Brasília. 2014.

5. BVS, Biblioteca Virtual em Saúde. Ministério da Saúde. Cálculo de doses de álcool. Rede Telessaúde Brasil. s/ano. Disponível em: https://aps.bvs.br/apps/calculadoras/?page=8. Acesso em: 20 agost.2018.

6. BOGENSCHUTZ MP, et al. Brief Intervention for Patients With Problematic Drug Use Presenting in Emergency Departments A Randomized Clinical Trial. JAMA Intern Med. 2014. 174(11):1736-1745.

7. DAWSON-ROSE C, et al. Changes in Specific Substance Involvement Scores among SBIRT recipients in an HIV primary care setting. Addict Sci Clin Pract. 2017;12(1):34.

8. DIEPERINK E, et al. Efficacy of motivational enhancement therapy on alcohol use disorders in patients with chronic hepatitis C: a randomized controlled trial. Addiction. 2014. 109(11):1869-77.

9. FARIA JG e SCHNEIDER DR. O perfil dos usuários do Capsad-Blumenau e as políticas públicas em saúde mental. Psicologia \& Sociedade. 2009. 21 (3): 324-333. 
10. FIGUEIREDO MFS, et al. Modelos aplicados as atividades de educação em saúde. Rev Bras Enferm. 2010. 63(1):117-21.

11. GABATZ RIB, et al. Percepção do usuário sobre a droga em sua vida. Esc. Anna Nery. 2013. 17(3): 520-525.

12. GENTIL V. Drauzio Dichava \#2 - A brisa. [Série/ Entrevista] 22/04/2019. Disponível em: https://drauziovarella.uol.com.br/videos/series-e-documentarios/drauzio-dichava-2-a-brisa/. Acesso em: 31 agost.2019.

13. GLASS JE, et al. Rethinking alcohol interventions in health care: a thematic meeting of the International Network on Brief Interventions for Alcohol \& Other Drugs. Addict Sci Clin Pract. 2017.12(1):1-14.

14. FROTA GAS, et al. Experiência de Usuários Acerca do Uso de Drogas. Rev. Bras. Promoç. Saúde.2018. 31(3): 1-11.

15. LIMAEH, et al. A prática dos grupos reflexivos sobre drogas como estratégia possível para redução de riscos e danos. Pesqui. prát. psicossociais.2018.12(3): 1-17.

16. MALBERGIER A e JUNIOR-OLIVEIRA H. Transtornos relacionados ao uso de substancias. Cap. 14. In: KAPCZINSKI, $\mathrm{F}$ et al (ORG). Bases Biológicas dos Transtornos Psiquiátricos Uma abordagem Translacional. Editora: Artmed.2011.

17. MARTINS JS e MACRAE E. Por um Olhar sociocultural sobre a questão das drogas. In: Antônio Nery Filho, Andrea Leite Ribeiro Valério (organizadores). Módulo para capacitação dos profissionais do projeto consultório de rua. EDUFBA/UFBA.2010. 89 p.

18. MONTEIRO SFS, et al. Perfil Sociodemográfico e Adesão ao Tratamento de Dependente de álcool em CAPS AD do Piauí. Esc Anna Nery (impr.) 2011. 15 (1):90-95.

19. MORIN JG, et al. A Population-Based Analysis of the Relationship Between Substance Use and Adolescent Cognitive Development. Am J Psychiatry. 2019. 176(2):98-106.

20. NÓBREGA MPSS. Fenômeno das drogas lícitas e ilícitas e assistência de enfermagem. Cap. 13. In: CARVALHO, MB. (ORG). Psiquiatria para Enfermagem. Editora Ridel. 2012.p. 351

21. QUEIROS PJP, et al. Autocuidado: o contributo teórico de Orem para a disciplina e profissão de Enfermagem. Revista de Enfermagem Referência.2014. IV(3): 157-164.

22. OLIVEIRA EN, et al. O Cuidado Multiprofissional na Prevenção de Internações Relacionadas ao Uso de Crack. J. res.: fundam. care. Online. 2019. 11(5): 1293-1299.

23. OMS, Organização Mundial da Saúde. Relatório Global sobre Álcool e Saúde - 2018. Genebra, Sui\&am Relatório Global sobre Álcool e Saúde - 2018.

24. OREM DE e TAYLOR SG. Reflections on nursing practice science: the nature, the structure, and the foundation of nursing sciences Nurs Sci Q. 2011. 24(1):35-41.

25. RIGONI MS, et al. Consequências neuropsicológicas do uso da maconha em adolescentes e adultos jovens. Ciências \& Cognição.2006. 8 (1):118-126.

26. ROCHA THR, et al. A desinstitucionalização no contexto da reforma psiquiátrica e seus desdobramentos: um relato sobre práticas em um CAPS. Vínculo. 2019.16 (1):1-16.

27. SALCI MA, et al. Educação em Saúde e suas Perspectivas Teóricas: Algumas Reflexões. Texto Contexto Enferm. 2013. (1): 224-30.

28. SANTOS HA, et al, Educação em Saúde: uma estratégia no cuidado com idosos hipertensos. Pesquisa em Foco. 2018. 23(1):194-206.

29. SOUZA SEF, et al. Abordagem na rua as pessoas usuárias de substâncias psicoativas: um relato de experiência. Saúde Debate. 2017. 41(112): 331-339.

30. SILVA JVS, et al. Capacidades de Autocuidado e Sua Relação com os Fatores Condicionantes Básicos: Um Estudo em Unidades Básicas de Saúde. Revista Eletrônica Acervo Saúde. 2012.4 (1): 185-199.

31. SILVA JV e DOMINGUES EAR. Adaptação cultural e validação da escala para avaliar as capacidades de autocuidado. Arq. Ciênc. Saúde. 2017. 24(4): 30-36.

32. SILVA JV e KIMURA M. Adaptação Cultural e Validação do Instrumento de Capacidade de Autocuidado "Appraisal of self-care agency sacale". Trabalho de Pesquisa. (Doutorado em Enfermagem) - Escola de Enfermagem da Universidade de São Paulo, SP. 2002.352p.

33. SOUZA LPS, et al. Mudanças favorecidas pela educação em saúde na perspectiva dialógica. EF Deportes. Com. Revista Digital. 2011. 16 (16):1.

34. UNODC, United Nations office on Drugs and Crime. Relatório Mundial sobre Drogas 2019: 35 milhões de pessoas em todo o mundo sofrem de transtornos por uso de drogas, enquanto apenas uma em cada sete pessoas recebe tratamento.2019.

35. VASCONCELOS SC, et al. Demandas de Autocuidado em Grupo Terapêutico: Educação em saúde com Usuários de substâncias Psicoativas. Rev. enferm, 2013. 21(1):79-83.

36. ZARSHENAS L, et al. Anger management in substance abuse based on cognitive behavioral therapy: an interventional study. BMC psychiatry. 2017. 17(1): 375. 